Short communication

\title{
COMPARISON OF TWO BLOOD MEAL PRESERVATION METHODS FOR USE IN ELISA-BASED IDENTIFICATION OF BLOOD-FED GLOSSINA SPECIES
}

\author{
Mesfin Lulu, Dejene Tilahun and Tefera Asfaw \\ Ethiopian Health and Nutrition Research Institute \\ PO Box 1242, Addis Ababa, Ethiopia
}

\begin{abstract}
Laboratory reared Glossina morsitans morsitans were fed on immobilized Swiss Albino Mice in an attempt to compare delectability of blood meal preserved as filter paper smears or as whole intact dried specimens. Both techniques showed similar trends with regards to absorbance values. Moreover, the inclusion of $25 \%$ ethanol seems to have improved the detection of the blood meal in both cases. Bloodmeals preserved on filter paper deteriorate rapidly with time. Therefore it would be advantageous to preserve insects as dried whole specimens if the identification process is not performed immediately.
\end{abstract}

Key words/phrases: Blood meal, preservation, Glossina, ELISA

\section{INTRODUCTION}

Detection of bloodmeals in hematophagous insects, especially in those with public health importance, is particularly useful in understanding the epidemiology of vector-borne diseases and to design effective control strategies.

Insect specimens are primarily preserved in three ways for blood meal identification (Weitz, 1956; Boreham, 1976; Beier et al., 1988). The most widely used technique for preserving bloodmeals is by using filter paper (Edrissian and Hafizi, 1982). In this method the insect is dissected and the abdominal content is squeezed on to a filter paper and smeared evenly (Weitz, 
1956). The other methods are freezing (Burkot and Defoliat, 1982) and drying (Beier, et al., 1988) whole insect specimens.

Various studies have been conducted to evaluate different blood meal preservation procedures in mosquitoes. Temelis and Lofty (1963) have obtained good results by keeping insect specimens in gelatin capsules under freezing conditions as compared to filter papers using the precipitin test. Burkot and Defoliart (1982) have also obtained similar results by using ELISA on frozen specimens. The need for refrigeration however has limited the wide application of the freezing method (Edman and Schmid, 1970).

Filter paper smears and the drying method because of their simplicity, minimal space required for storage and ease of performance have enjoyed wider application. The present work attempts to communicate the advantages of the drying method as compared to the filter paper technique in the detection of bloodmeals in tsetse flies using ELISA.

\section{Sample preparation}

\section{MATERIALS AND METHODS}

Laboratory reared Glossina morsitans morsitans were fed on immobilized Swiss Albino Mice. They were subsequently killed 2, 4, 6, 8, 48 and 96 hours after blood ingestion. In each category specimens were divided in two groups. In the first group, tsetse were air dried and kept in vials, in the other group the gut contents were smeared on filter papers. Each category consisted of 14-26 specimens. In both cases samples were kept at room temperature $\left(21-25^{\circ} \mathrm{C}\right)$ until ELISA was performed.

Ten microlitre of human and mice blood was also applied separately onto filter paper strips and kept at room temperature. ELISA was performed on eight duplicates each, every two weeks, to observe the effect of storage on the delectability of blood samples.

\section{Blood meal identification}

Abdominal parts were dissected from the head-thorax portions of the dried tsetse specimens using dissecting needles. Abdominal parts were ground on porcelain dish in $50 \mu \mathrm{l}$ phosphate buffered saline (PBS) using a pestle. Ten 
microlitre of the homogenate was diluted in PBS containing 25\% ethanol (1:20) and $50 \mu \mathrm{l}$ of the diluent was added to flat bottomed 96 well microlitre plates (Linbro, USA). Similarly, dried spots of tsetse on filter paper were cut out as small paper discs ( $6 \mathrm{~mm}$ diameter) and eluted in $100 \mu \mathrm{l}$ PBS for 90 minutes at room temperature. Ten microlitre was then taken and the steps used for the dried specimens were repeated hereafter. The same procedure was followed for the detection of $10 \mu \mathrm{l}$ of mice and human blood spotted on filter papers.

Direct ELISA was then performed following the method of Beier et al. (1988), whereby microtitre plates were incubated overnight. Plates were then washed twice with PBS containing $0.5 \%$ Tween 20 (Sigma Chemicals, USA). Fifty microlitre of peroxidase conjugated anti-mouse IgG that was diluted to 1:2000 in $0.5 \%$ boiled casein containing $0.0025 \%$ Tween 20 was added to each well. The plate was then incubated at room temperature for $1 \mathrm{hr}$. Unbounded antimouse IgG was then removed by washing three times. One hundred microlitre of peroxidase substrate, ABTs [2,2'azino-di(3-ethylbenzthiazoline sulfate (6)] was then added to each well.

Absorbance was finally recorded at $405 \mathrm{~nm}$ using an ELISA reader (Labsystems Multiscan MCC 1340, Finland). Samples were considered positive when their absorbance values were greater than the mean plus three times the standard deviation of four negative controls, in this case unfed tsetse flies.

\section{RESULTS}

The direct ELISA was used to detect bloodmeals of tsetse flies from filter paper and from whole dried specimens. Both methods showed similar trends with a statistically significant correlation with regards to absorbance values $(r=0.87$, $\mathrm{P}<0.001$ ) for successive hours post ingestion (Fig. 1).

The overall percentage of positively reacting samples in both situations (Table 1) showed a very similar trend in which positivity sharply decreased after 48 hours post ingestion in both methods. Positive reactions were obtained for all tested specimens until $\mathbf{4 8}$ hours post ingestion in the filter paper smears, $\mathbf{4 1 \%}$ (7/17) of the smears showed positive reaction at 96 hours post ingestion. Similarly, for the dried samples, all tested sample showed positive reactions at 
2 and 6 hours post ingestion, 82\% (14/17) and 24\% (4/17) positive reactions were observed at 48 and 96 hours, respectively.

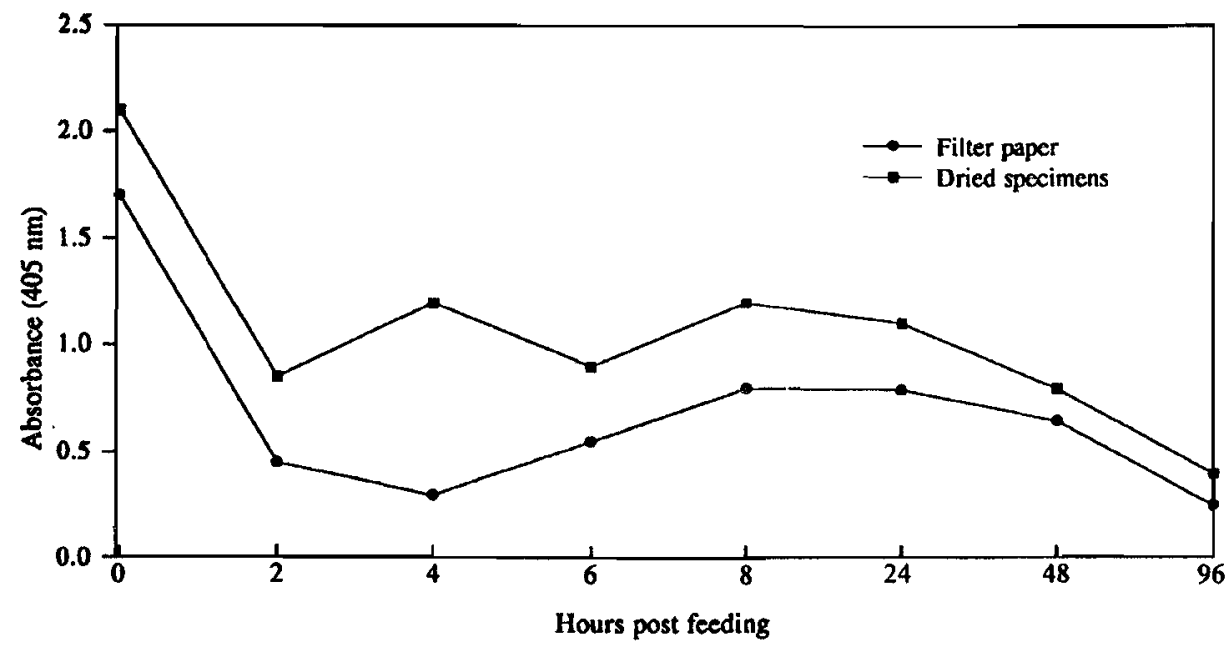

Fig. 1. Mean absorbance values of filter paper and dry specimens as determined by ELISA.

Table 1. Percentage positivity of filter paper smears and dried tsetse specimens as determined by ELISA.

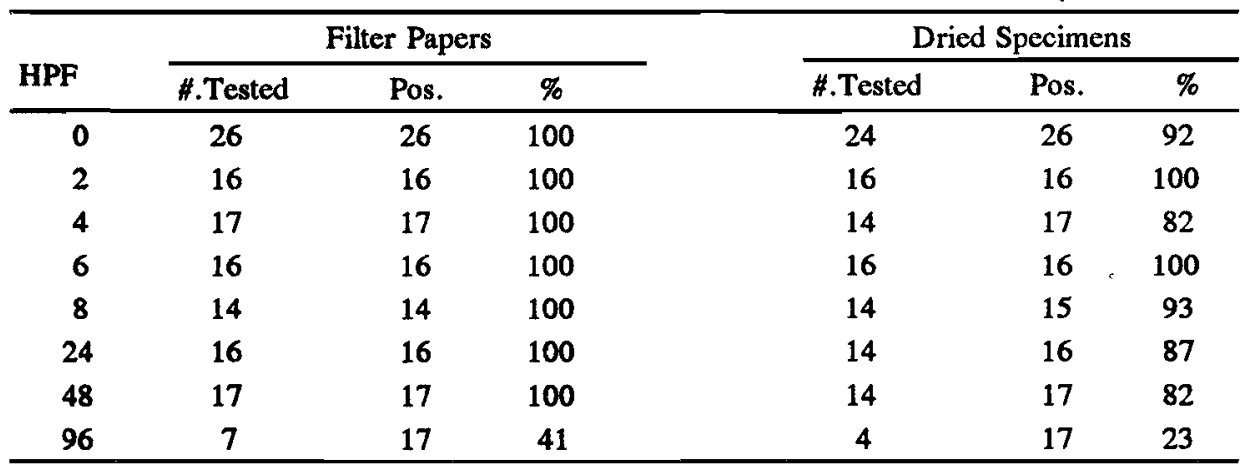

HPF, Hours Post Feeding. 
Steadily decreasing absorbance readings were observed with time for $10 \mu \mathrm{l}$ of mice blood on filter paper tested every two weeks (Fig. 2). In the case of an equal amount of human blood prepared in the same manner, a wide difference was witnessed in the absorbance values.

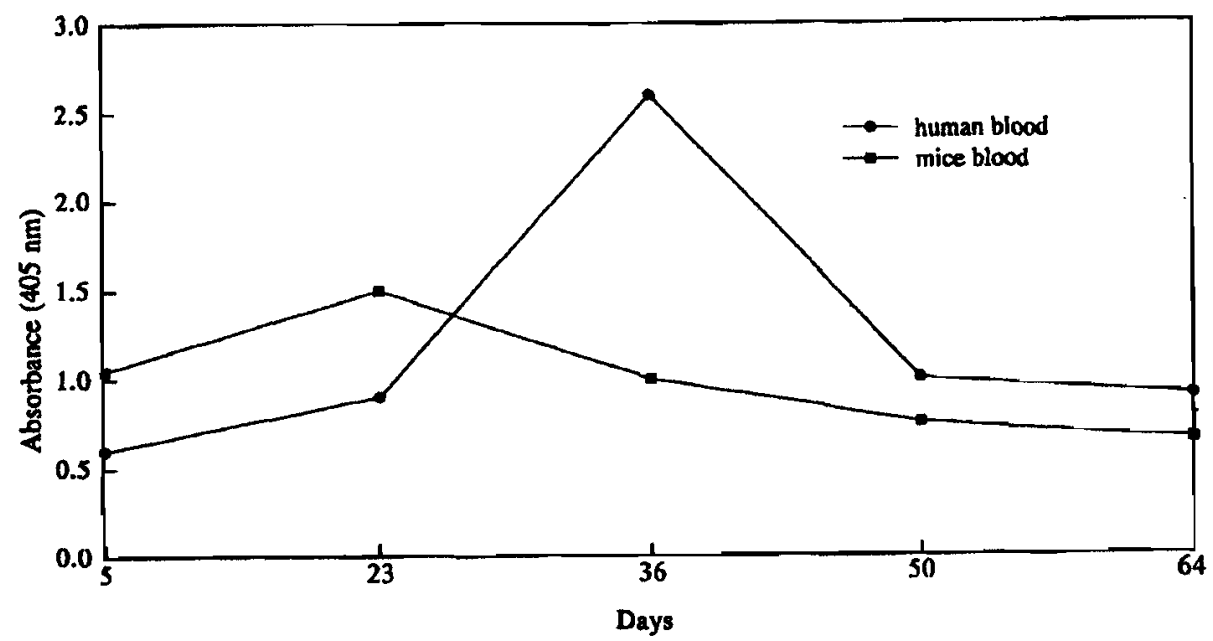

Fig. 2. Mean absorbance values of human and mice blood on filter paper, tested by ELISA every two weeks.

\section{DISCUSSION}

Field investigators have always opted for a simple, direct and uncomplicated means of preserving bloodmeals in insects for blood meal analysis. Because of these features both the filter paper smears and drying whole insect specimens have been used widely.

In this study attempts have been made to compare the two methods. Both methods have shown similar trends in absorbance values. The addition of $25 \%$ ethanol has improved the delectability of the blood meal in both cases. Beier, et al., (1988) have detected bloodmeals 23 and 32 hours post ingestion in frozen 
and dried mosquito specimens. Similarly, Edrissians and Hafizi (1982) reported the detection of bloodmeals of mosquitoes after 24 hours post ingestion. In this study $100 \%(17 / 17)$ of the bloodmeals on filter papers and $82 \%(14 / 17)$ of the bloodmeals in dried specimens were detected at 48 hours post ingestion. Even though it may be argued that the amount of the blood meal in tsetse flies is somewhat large as compared to mosquitoes and hence can be detected more easily, the high digestion rate of tsetse flies is known to compensate for the volume difference in bloodmeals (Weitz, 1956). The improvement in sensitivity by the addition of $25 \%$ ethanol may be due to the removal of insect fat body that could interfere with the detection process.

Bloodmeals smeared on filter paper have been extensively used due to their simplicity and because several smears from different insect samples can be made on one filter paper alone. However, serum proteins are known to rapidly deteriorate on filter paper (Tempelis and Lofty, 1963) and also the means of expressing the blood onto the filter paper is known to affect the outcome of the blood meal test (Beier, et al., 1988). Edman and Schmid (1970) have reported that optimum results for blood meal detection are obtained when specimens are dried as compared to other means of preservation. Similarly, an erratic and successively decreasing absorbance readings were observed in this study as time elapsed for human and mice blood expressed on filter paper respectively. Therefore, unless these filter papers are processed right away the results obtained from them would deteriorate with time.

In conclusion, we would like to communicate that bloodmeals of tsetse flies can be preserved as whole dried specimens and can produce results that are comparable to filter paper smears. Blood meal expressed as smears on filter paper produce unreliable results especially if they are not analyzed immediately. It would be therefore advantageous to preserve intact tsetse specimens dried and packed in vials for blood meal analysis instead of making filter paper smears as is often the case with tsetse flies.

\section{ACKNOWLEDGEMENT}

The authors wish to thank Ato Yared Makonnen for his technical help in this study. 


\section{REFERENCE}

1. Beier, J.C., Perkins, P.V., Wirtz, R.A., Koros, J., Diggs, D., Gargan, T.P. and Koech, D.K. (1988). Bloodmeal identification by direct enzyme-linked immunosorbent assay (ELASA), tested on Anopheles (Diptera: Culicidae) in Kenya. $J$. Med. Ento. 25:2-16.

2. Boreham, P.F.L. (1976). Sterilization of arthropod bloodmeals prior to bloodmeal identification. Mosquito News. 36:454-457.

3. Burkot, T.R. and Defoliat, G.P. (1982). Bloodmeal sources of Aedes triseriatus and Aedes vexans in southern Wisconsin forest endemicfor La Crosse encephalitis virus. Am. J. Trop. Med. Hyg. 3:376-381.

4. Edman, J.D. and Schmid, A.A. (1970). Comparison of precipitin-test results with mosquito bloodmeals after prolonged storage under different conditions. J. Med. Ento. 7:619-621.

5. Edrissian, G.H. and Hafizi, A. (1982). Application of enzyme-linked immunosorbent assay (ELISA) to identification of Anopheles mosquito bloodmeals. Trans. R. Soc. Trop. Med. Hyg. 76:54-56.

6. Tempelis, C.H. and Lofty, M.F. (1963). A modified precipitin method for identification of mosquito bloodmeals. Am. J. Trop. Med. Hyg. 12:825-831.

7. Weitz, B. (1956). Identification of bloodmeals of blood-sucking arthropods. Bull. WHO. 15:473-490. 